only provided risk factors leading to SIDS. Though avoidance of these risk factors has led to substantial reduction $(80 \%)$ in the rate of SIDS over last 2 decades, it is still the leading cause of death in infants between four weeks and 6 months of age. The most recent data provided by Irish central statistics office reports 14 deaths (0.21 deaths per 1000 live births) in year 2014. We aimed to find out the awareness of the risk factors leading to SIDS amongst mothers who delivered at Mullingar Regional Hospital.

Methods A prospective assessment was performed by distributing multidimensional questionnaire to the women admitted in postnatal ward following birth of their baby in Mullingar Regional Hospital, Ireland. Anonymously collected data was analysed using SPSS2 software. Regional hospital ethical committee approved the study.

Results One hundred two participants were included in analysis. 9.6 percent $(n=9)$ participants had never heard of SIDS. Of those who had, $46 \%$ (43/93) and 54\% (53/93) have heard it from healthcare providers and media respectively. Major risk factors identified by number of participants were; prone sleeping position $(80 \%, 75 / 93)$, overheating of the baby (91\%, 85/93), Soft bedding (90\%, 84/93), bed sharing of infants $(69 \%, 65 / 93)$, Maternal smoking during pregnancy (60\%, 56/93), Smoking around babies environment (58\%, $54 / 93$ ) and maternal alcohol intake during pregnancy $(56 \%$, 53/93). 20\% participants failed to identify prone sleeping position as the risk factor. A significant number of participants were unaware of other major risk factors such as smoking during pregnancy, smoking around baby and bed sharing with baby. $100 \%$ of the participants wanted more information about SIDS. Televised campaign (68\%, 70/102) and reading materials $(56 \%, 58 / 102)$ were the 2 most preferred method of delivering SIDS information to the participants. $66 \%(68 / 102)$ participants wanted the information delivered as part of prenatal education and further 29\% (30/ 102) wanted it before discharge from the post-natal ward. $53.9 \%(55 / 102)$ and $31 \%(32 / 102)$ participants opted midwife/maternity nurses and paediatrician respectively as the person to deliver information about SIDS.

Conclusions This study concludes that there is a wide gap in knowledge about risk factors for SIDS and almost all participants felt they need more information. We hope implementing methods to narrow this knowledge gap would further reduce the incidence of SIDS.

\section{OC33 ALTERED TOLL LIKE RECEPTOR 2 (TLR2) SIGNALLING IN CHILDREN WITH DOWN SYNDROME}

\footnotetext{
1,2,3 Dean Huggard* ${ }^{1}$ Wen J Kaoy, ${ }^{3}$ Fiona McGrane, ${ }^{1,3}$ Edna Roche, ${ }^{1,3}$ Joanne Balfe, ${ }^{3}$ Niamh Lagan, ${ }^{1,3}$ Emer Ryan, ${ }^{4} \mathrm{~T}$ Ronan Leahy, ${ }^{5}$ Orla Franklin, ${ }^{2}$ Derek Doherty, ${ }^{1,3,6}$ Eleanor Molloy. ${ }^{1}$ Paediatrics, Trinity College, the University of Dublin, Dublin, Ireland; ${ }^{2}$ Trinity Translational Medicine Institute (TTMI), Trinity College Dublin, Dublin, Ireland; ${ }^{3}$ Paediatrics, Tallaght Hospital, Dublin, Ireland; ' Immunology, Our Lady's Children's Hospital, Crumlin, Dublin, Ireland; ${ }^{5}$ Cardiology, Our Lady's Children's Hospital, Crumlin, Dublin, Ireland; ${ }^{6}$ Neonatology, Our Lady's Children's Hospital, Crumlin, Dublin, Ireland
}

10.1136/archdischild-2019-epa.32

Background Toll like receptors (TLRs) are key in initiating innate immune responses. TLR2 is crucial in recognising lipopeptides from gram positive bacteria and is implicated in chronic inflammation. Children with Down syndrome (DS) are prone to infections from these pathogens and have an increased risk of autoimmunity. Sparstolonin $\mathrm{B}(\mathrm{SsnB})$ is a
TLR antagonist shown to reduce cytokine production and improve outcomes in sepsis. We hypothesized that TLR2 signalling may be anomalous in children with DS and contribute to their clinical phenotype.

Aims We aimed to evaluate TLR2 pathways in 3 ways; by determining the expression of TLR2 on the surface of neutrophils, monocytes, and their subsets; examine gene expression of key regulatory proteins involved in TLR signal propagation, MyD88, IRAK4, and TRIF; and lastly to determine cytokine production at baseline and following immunomodulation with pro-inflammatory stimuli (LPS, Pam3Csk4) and the anti-inflammatory agent SsnB.

Methods Whole blood was collected from children with DS and age matched controls. Samples were treated with lipopolysaccharide (LPS) $10 \mathrm{ng} / \mathrm{ml}$, Pam3Csk4 $(5 \mathrm{ng} / \mathrm{ml})$, SsnB $(10 \mu \mathrm{M})$ or in combination. TLR2 and CD11b expression on neutrophils and monocytes was evaluated by flow cytometry. RNA was isolated from Trizol $^{\circledR}$, cDNA was synthesized and then evaluated by quantitative PCR for expression of MyD88, IRAK4, and TRIF. A panel of pro and anti-inflammatory cytokines were evaluated using the MSD ${ }^{\circledR}$ MULTI-SPOT assay system from Mesoscale (MSD Diagnostics, USA). Statistical analysis employed unpaired t-tests, ANOVA, analysed using GraphPad Prism and FloJo software.

Results Children with DS $(n=20)$ and controls $(n=15)$ were recruited. TLR2 expression was significantly raised on neutrophils $(p=0.02)$, total monocytes $(p=0.05)$, intermediate monocytes $(p=0.02)$ in children with DS compared to controls. At baseline the expression of MyD88 was significantly lower $(\mathrm{p}=0.001)$, and TRIF significantly raised in children with DS $(\mathrm{p} \leq 0.0001)$. The TLR antagonist $\mathrm{SsnB}$ was effective at reducing TLR2 and CD11b expression and abrogating cytokine production in both cohorts.

Conclusion TLR2 pathway is dysregulated in DS. There is greater expression of TLR2 on the surface of neutrophils and monocytes. Downstream signalling is altered with reduced MyD88 and increased expression of TRIF, which may represent compensatory upregulation of MyD88 independent pathways. This altered innate immunity may contribute to chronic inflammation in DS. SsnB attenuates pro-inflammatory mediators and could be of therapeutic benefit.

\section{OC34 EVALUATING THE QUALITY OF HIP SURVEILLANCE RADIOGRAPHS IN CHILDREN WITH CEREBRAL PALSY}

${ }^{1}$ Miriam Nyeko-Lacek*, ${ }^{2}$ Clare Carpenter. ${ }^{1}$ Cardiiff University, Cardiff, UK; ${ }^{2}$ University Hospital of Wales, Cardiff, UK

\subsection{6/archdischild-2019-epa.33}

Background Hip dislocation can seriously affect the quality of life of children with cerebral palsy. Regular radiological surveillance is an important part of the management of cerebral palsy, but can be challenging, due to the nature of the condition.

Objectives To evaluate the technical quality of hip surveillance radiographs of children with cerebral palsy and to assess the suitability of the 'Hip Screen' phone application to be used in measurement of migration percentage.

Materials and methods 100 radiographs of patients undergoing hip surveillance due to a risk of hip dislocation caused by cerebral palsy. Pelvic rotation and inclination were assessed using the standards recommended by the literature. Migration 
percentage was calculated 1) using the IMPAX measurement tools 2) by using the 'Hip Screen' phone application and 3) using the radiology report. The three methods were compared.

Results The technical quality of most of the hip radiographs was clinically acceptable. There was a lower percentage of radiographs with adequate pelvic inclination than those with adequate pelvic rotation. There was good agreement between measurements taken with the app, measurements taken directly on IMPAX and the radiology report.

Conclusions Technical quality of the hip radiographs evaluated is generally sufficient to assess hip migration. The 'Hip Screen' phone application can be considered a reliable method of hip surveillance.

Discussion Using the 'Hip Screen' phone application may be more acceptable to future clinicians as may can be readily accessed and may be a useful tool for educating families of children with cerebral palsy.

\section{OC35 PULSE WAVE VELOCITY, FREE FATTY ACIDS, AND STANDARD LIPIDOGRAM IN NORMAL WEIGHT, OVERWEIGHT AND OBESE SCHOOL CHILDREN}

${ }^{1}$ Bernarda Vogrin*, ${ }^{2,3,4}$ Marjan Slak Rupnik, ${ }^{5}$ Dušanka Mičetić-Turk. ${ }^{1}$ PEDENJPED D.0.0., Lenart, Slovenia; ${ }^{2}$ Institute of Physiology, Medical faculty, University of Maribor, Maribor, Slovenia; ${ }^{3}$ Center of physiology and pharmacology, Medical University Vienna, Vienna, Austria; ${ }^{4}$ Alma Mater Europaea - European Center Maribor, Maribor, Slovenia; ${ }^{5}$ Medical faculty, University of Maribor, Maribor, Slovenia

\subsection{6/archdischild-2019-epa.34}

Background and Aim Childhood obesity poses a serious threat for the development of metabolic syndrome, diabetes and cardiovascular diseases (CVD) later in life. Nowdays we are able to recognize metabolic and vascular pathology at a discrete, completely curable stage and long before clinical signs of metabolic syndrome and CVD. Increased arterial stiffness is a good indicator of initial intimal atherosclerotic lesions, easily detected with measuring of pulse wave velocity (PWv) and augmentation index (AIx). Atherosclerosis is initiated and promoted by disturbed lipid metabolism. The increased level of serum free fatty acids (FFA) is supposed to be an early sign of imminent metabolic disturbance. The aim of our study was to assess and compare the metabolic and arterial health in a sample of normal weight, overweight and obese school children from north-west Slovenia.

Materials and Methods 81 healthy, metabolic syndrome free school children aged 11-16 years participated in our study. According to the standard deviation score of body mass index (SDS BMI), the underweight represented $1(1.2 \%)$, the normal weight 38 (46.9\%), the overweight 22 (27.2\%)and the obese $20(24.7 \%)$ of the sample. The AIx, PWv, free fatty acids, standard lipidogram and serum glucose (GLU) were recorded.

Results In the group of overweight children (SDS BMI 1-2) compared to normal weight (SDS BMI -2 to 1) no differences in arterial nor metabolic health parameters were recorded. In the obese children (SDS BMI >2) a significantly hiegher PWv $(\mathrm{p}<0.01)$ triglycerides $(\mathrm{TRG}) \quad(\mathrm{p}<0.01)$, the ratio between TRG and hiegh density lipoproteins (HDL) $(\mathrm{p}<0.001)$, the TRG/low density lipoproteins (LDL) $(p<0.01)$, FFA $(p<0.05)$ and lower HDL $(p<0.01)$ than in normal weight were found.

Conclusion Despite the detected increased arterial stiffness of aorta and signs of metabolic disturbance, the obese children were still free of clinical signs of metabolic syndrome. Our results could be important for planning of preventive and therapeutic meassures of overweight and obese children. According to this study, preventive activities should be provided to the overweight children, to maintain their arterial and metabolic health, while obesity has to be cured promptly to recover the metabolic stability and to cure initial atherosclerotic changes.

\section{OC36 THE UTI-UTI AUDIT: UNDERSTANDING THE TREATMENT OF INFANTS WITH URINARY TRACT INFECTION}

Warren McCue*, Laura Bingham, Lesley-Ann Funston, Shilpa Shah. Craigavon Area Hospital, Craigavon, UK

10.1136/archdischild-2019-epa.35

Background and aims In infants with UTI, practice previously was to commence prophylaxis and investigate for underlying renal pathology. DMSA and MCUG scans have associated risks, and along with urgent inpatient ultrasound, they represent a burden on radiology services. Antibiotic prophylaxis has been linked with rising bacterial resistance.

The current NICE guideline on UTIs in children sets clearer indications for investigation, recommending that only infants with atypical features or recurrence should undergo DMSA and MCUG. Are these recommendations being followed in the youngest infants (0-3 months) diagnosed with UTI?

Methods A retrospective NIECR (Northern Ireland Electronic Care Record) audit was performed on all infants under 3 months diagnosed with UTI in Craigavon Hospital from January - December 2017. Data compared to NICE recommendations and analysed.

Results Data collected for 31 admissions, representing 29 patients: $66 \%$ male, $34 \%$ female.

$55 \%$ classified 'typical', 45\% 'atypical', though $68 \%$ of all cases underwent DMSA/MCUG.

93\% of atypical UTIs were managed as per NICE, compared with only $24 \%$ of typical UTIs. Of the remaining typical cases, 6\% were underinvestigated and 71\% were overinvestigated: $41 \%$ with urgent inpatient US plus DMSA/ MCUG, 30\% with urgent inpatient US alone.

$77 \%$ of atypical cases had significant abnormalities on scans. Only two overinvestigated typical cases, or $17 \%$, showed significant abnormality. However, one had a firstdegree family history of vesicoureteric reflux. This is a recognised NICE atypical feature in older age-groups but is not listed for the $<6$ month group.

Conclusion Atypical UTIs were managed more appropriately than typical UTIs when compared against NICE recommendations. As expected from the evidence base, atypical and recurrent UTIs were commonly associated with underlying renal abnormalities. Typical UTIs, when investigated, were rarely associated with underlying abnormalities or scarring. A laminated aide-mémoire with appropriate investigations is now available in clinical areas on children's ward for quick recall. 